\title{
Correlação entre o consumo e a deposição de ácidos graxos em bovinos suplementados com glicerina de baixa pureza em pastagens
}

\section{Correlation between consumption and deposition of fatty acids in cattle supplemented with low purity glycerin in pastures}

\author{
Evani Souza de Oliveira Strada ${ }^{1 *}$; Robério Rodrigues Silva ${ }^{2}$; \\ Gleidson Giordano Pinto de Carvalho ${ }^{3}$; Larissa Pires Barbosa ${ }^{1}$; \\ Fabiana Lana de Araújo ${ }^{1}$; Alexandre Moraes Pinheiro학 Fabiano Ferreira da Silva²; \\ Kaliane Nascimento de Oliveira ${ }^{4}$; Carlos Emanuel Eiras ${ }^{4}$
}

\begin{abstract}
Resumo
Objetivou-se determinar a correlação linear existente entre os ácidos graxos consumidos e depositados no músculo Longissimus dorsi de 35 bovinos machos não castrados, mestiços, com predominância da raça Nelore, com peso inicial médio de 428,0 $\pm 32,11 \mathrm{~kg}$, terminados em pastagem de Brachiaria decumbens $\mathrm{cv}$. Basilisk. O delineamento utilizado foi inteiramente casualizado, com cinco dietas e sete animais por dieta durante 74 dias. As dietas consistiram de níveis de inclusão da glicerina $(0,3,6,9$ e $12 \%$ ) na matéria seca (MS). Os animais foram pesados a cada 28 dias para avaliação do ganho de peso médio diário (GMD) e ajuste das dietas. Amostras do capim Brachiaria decumbens, dos suplementos, assim como do músculo Longissimus dorsi foram analisadas para avaliação do perfil de ácidos graxos. A correlação foi realizada estimando-se os coeficientes de correlação linear de Pearson. Os resultados encontrados demonstram a existência de correlações entre os ácidos graxos consumidos com aqueles depositados no músculo dos animais. As correlações observadas modificaram o perfil dos ácidos graxos da carne com redução da concentração dos ácidos graxos monoinsaturados e aumento da concentração dos ácidos graxos da série $\omega-6$, assim como da razão entre os ácidos graxos da série $\omega-6$ e $\omega$ - 3. Não foram verificadas correlações com os ácidos graxos hipercolesterêmicos (láurico, miristico e palmítico) do músculo dos bovinos suplementados com glicerina de baixa pureza.
\end{abstract}

Palavras-chave: Biodiesel, bovinos de corte, glicerol, lipídios, pasto

\begin{abstract}
The objective was to determine the existing linear correlation between the consumed fatty acids and deposited in the muscle Longissimus dorsi 35 uncastrated male bovine animals, crossbred Nellore predominance, with average weight of $428.0 \pm 32.11 \mathrm{~kg}$, ending in Brachiaria decumbens cv. Basilisk. The experimental design was completely randomized with five diets and seven animals for 74 days. Diets consisted of glycerin inclusion levels $(0,3,6,9$ and 12\%) in the dry matter (DM). The animals
\end{abstract}

\footnotetext{
${ }^{1}$ Profs., Universidade Federal do Recôncavo da Bahia, UFRB, Cruz das Almas, BA. E-mail: evanistrada@ufrb.edu.br; 1pires73@ yahoo.com.br; fabianalanadearaujo@hotmail.com; amp@ufrb.edu.br

2 Profs., Universidade Estadual do Sudoeste da Bahia, UESB, Itapetinga, BA. E-mail: rrsilva.uesb@hotmail.com; ffsilva@cnpq. com.br

3 Prof., Universidade Federal da Bahia, UFBA, Salvador, BA. E-mail: gleidsongiordano@yahoo.com.br

${ }^{4}$ Discentes do Curso de Doutorado do Programa de Pós-Graduação em Zootecnia, Universidade Estadual de Maringá, UEM, Maringá, PR. E-mail: kalyoliveira@hotmail.com; carlos.eiras@hotmail.com

* Autor para correspondência
} 
were weighed every 28 days to assess the weight gain (ADG) and adjusts the diets. Samples of Brachiaria decumbens, supplements, and Longissimus dorsi were analyzed for evaluation of fatty acid profile. The correlation was performed by estimating the coefficients of linear correlation. The observed correlation profile of the modified meat fatty acid with a reduced concentration of monounsaturated fatty acids and increasing the concentration of fatty acids of the $\omega$ series -6 as well as the ratio between the fatty acids of the series $\omega$ - and $\omega 6-3$. No correlations were found with hipercolesterêmicos fatty acids ( lauric , myristic and palmitic ) of bovine muscle supplemented with low purity glycerin .

Key words: Biodiesel, beef cattle, glycerol, lipids, pasture

\section{Introdução}

O uso de alimentos alternativos pode ser uma escolha viável na produção de ruminantes, principalmente se não comprometerem 0 desempenho do animal e se seus custos forem menores que o dos alimentos convencionais (GUNN et al., 2010).

Muitas pesquisas têm sido desenvolvidas com a glicerina bruta, coproduto da indústria do biodiesel, como uma alternativa na alimentação de ruminantes (EIRAS et al., 2014; LAGE et al., 2010; MACH; BACH; DEVANT, 2009; PARSONS; SHELOR; DROUILLARD, 2008; WANG et al., 2009). Para cada tonelada de biodiesel, são produzidos aproximadamente $100 \mathrm{~kg}$ de glicerina bruta (OLIVEIRA et al., 2013).

O glicerol, principal componente da glicerina, é energético e tem potencial de uso como substituto parcial dos grãos de cereais ou outros ingredientes com elevado teor de amido (DEFRAIN et al., 2004; LAMMERS et al., 2008).

Quando absorvido diretamente pelo epitélio ruminal, o glicerol é direcionado ao fígado onde, pela ação da enzima glicerol quinase, é transformado em glicose. Contudo, quando fermentado no rúmen gera propionato que, no fígado por meio do ciclo de Krebs, é metabolizado a oxaloacetato, que pode ser utilizado na síntese da glicose pela via gliconeogênica (LAGE et al., 2010) ou, em condições de energia excedente, é direcionado para a síntese de triacilgliceróis (PALMQUIST ; MATTOS, 2006).
A taxa de triacilgliceróis, assim como o perfil de seus ácidos graxos são os principais atributos da carne avaliados pelos consumidores. Esse fato deve-se à divulgação de resultados de estudos que evidenciam correlação positiva entre a gordura saturada e o colesterol com problemas cardiovasculares, obesidade e câncer (DEPARTMENT OF HEALTH, 1994; GRUNDY; DENKE, 1990).

Entretanto, ainda existem muitas controvérsias com relação ao real efeito da gordura animal sobre a saúde humana (BROUWER; WANDER; KATAN, 2013; GEBAUER et al., 2011). Desta forma, enquanto não há um consenso entre a comunidade cientifica, a recomendação é que seja reduzida a ingestão de gorduras com altas concentrações de colesterol e de ácidos graxos saturados e ácidos graxos trans e aumento do consumo de ácidos graxos monoinsaturados e dos ácidos graxos poliinsaturados, uma vez que estes estão associados à diminuição das doenças cardiovasculares (COSTA et al., 2008).

Segundo Geay et al. (2001), a quantidade e a natureza dos lipídios encontrados na carne são altamente variáveis, dependentes das condições de alimentação, da digestão, da absorção intestinal, do metabolismo hepático e do sistema de transporte desses lipídios.

Assim, objetivou-se avaliar a correlação linear entre os ácidos graxos da dieta contendo teores de inclusão de glicerina de baixa pureza e do músculo Longissimus dorsi de bovinos machos, terminados em pastagem de Brachiaria decumbens. 


\section{Material e Métodos}

O experimento foi conduzido no Setor de Bovino de Corte da Universidade Federal do Recôncavo da Bahia (UFRB), campus de Cruz das Almas $\mathrm{BA}$, em uma área de 35 hectares (ha) formada de Brachiaria decumbens, dividida em cinco piquetes com, aproximadamente, sete ha cada, com acesso a praça de alimentação.

O período experimental compreendeu os meses de setembro a dezembro de 2010, num total de 88 dias, sendo os primeiros 14 dias destinados à adaptação dos animais ao manejo e as dietas experimentais e 74 dias para coletas dos dados.
Utilizou-se 35 bovinos machos não castrados, mestiços, com predominância da raça Nelore, com peso corporal inicial médio de 428,0 $\pm 32,11$ $\mathrm{kg}$, suplementados na proporção de $1,0 \%$ do peso corporal (PC), distribuídos em um delineamento inteiramente casualizado, com cinco dietas e sete animais por dieta.

Os suplementos continham $0,3,6,9$ e $12 \%$ de inclusão de glicerina de baixa pureza na dieta total, sendo formuladas segundo o NRC (2000) para atender as exigências de mantença e ganho de peso médio diário (GMD) de $1,2 \mathrm{~kg} \mathrm{dia}^{-1}$ (Tabela 1) e fornecidas uma vez ao dia, às 11 horas.

Tabela 1. Composição percentual dos suplementos com base na matéria seca e composição química dos suplementos e pasto ofertados.

\begin{tabular}{|c|c|c|c|c|c|c|}
\hline & \multirow{2}{*}{$\begin{array}{l}\text { Brachiaria } \\
\text { decumbens }\end{array}$} & \multicolumn{5}{|c|}{ Níveis de inclusão de glicerina de baixa pureza (\%MS) } \\
\hline & & 0 & 3 & 6 & 9 & 12 \\
\hline \multicolumn{7}{|l|}{ Ingredientes (\%) } \\
\hline Milho Grão Moído & & 79,35 & 70,91 & 62,30 & 53,53 & 44,58 \\
\hline Farelo de Soja & & 17,30 & 18,95 & 20,64 & 22,35 & 24,10 \\
\hline Glicerina bruta & & - & 6,76 & 13,65 & 20,68 & 27,85 \\
\hline Ureia & & 1,85 & 1,87 & 1,88 & 1,90 & 1,92 \\
\hline Mistura Mineral $^{1}$ & & 0,99 & 1,00 & 1,00 & 1,02 & 1,02 \\
\hline Calcário & & 0,51 & 0,51 & 0,52 & 0,52 & 0,53 \\
\hline Total & & 100,00 & 100,00 & 100,00 & 100,00 & 100,00 \\
\hline \multicolumn{7}{|l|}{ Componentes (\%) } \\
\hline Matéria seca & 31,60 & 85,81 & 87,52 & 86,00 & 84,49 & 85,36 \\
\hline Matéria mineral & 8,30 & 3,06 & 3,30 & 3,53 & 3,49 & 3,81 \\
\hline Matéria orgânica & 91,70 & 96,94 & 96,70 & 96,47 & 96,51 & 96,19 \\
\hline Proteína bruta & 13,10 & 24,39 & 24,69 & 24,29 & 22,33 & 23,54 \\
\hline Extrato etéreo & 2,98 & 3,13 & 5,39 & 6,23 & 6,62 & 8,56 \\
\hline Fibra em detergente neutro & 69,61 & 25,49 & 25,01 & 25,25 & 18,98 & 18,51 \\
\hline $\mathrm{FDNcp}^{2}$ & 62,32 & 13,64 & 7,41 & 5,87 & 6,33 & 4,28 \\
\hline Carboidratos totais & 75,55 & 69,42 & 66,62 & 65,95 & 67,56 & 64,09 \\
\hline $\mathrm{CNFcp}^{3}$ & 5,94 & 43,93 & 41,61 & 40,70 & 48,58 & 45,58 \\
\hline $\mathrm{NDT}^{4}$ & 56,87 & 89,00 & 85,84 & 84,87 & 92,13 & 92,66 \\
\hline $\mathrm{CEM}^{5}(\mathrm{Mcal}$ de EM/kg de MS) & 3,88 & 3,92 & 3,78 & 3,74 & 4,06 & 4,09 \\
\hline
\end{tabular}

${ }^{1}$ Composição em 100g: Cloreto de sódio $(\mathrm{NaCl})$ - 47,15g; Fosfato bicálcico - 50g; Sulfato de zinco - 1,5g; Sulfato de cobre- 0,75g; Sulfato de cobalto - 0,05g; Iodato de potássio - $0,05 \mathrm{~g}$; Sulfato de magnésio- $0,5 \mathrm{~g}$. ${ }^{2}$ Fibra em detergente neutro corrigida para cinzas e proteínas; ${ }^{3}$ Carboidratos não fibrosos corrigidos para cinzas e proteínas; ${ }^{4}$ Nutrientes digestíveis totais $;{ }^{5}$ Concentração de energia metabolizável.

Fonte: Elaboração dos autores. 
Os animais foram pesados a cada 28 dias, sempre após um jejum alimentar de 14 horas, para acompanhamento do desempenho animal e ajuste da dieta.

As análises químicas das amostras da forragem e dos suplementos (Tabela 1) foram realizadas no laboratório de Nutrição Animal da Universidade Estadual do Sudoeste da Bahia em ItapetingaBA. Foram avaliados os teores de matéria seca (MS), massa orgânica (MO), proteína bruta (PB), extrato etéreo (EE), segundo técnicas descritas por Silva e Queiroz (2002). Para a determinação da fibra em detergente neutro (FDN) utilizou-se a metodologia proposta por Van Soest, Robertson e Lewis (1991).

Os carboidratos totais (CHOT) estimados segundo Sniffen et al. (1992), como:

$$
\mathrm{CHOT}=100-(\% \mathrm{~PB}+\% \mathrm{EE}+\% \text { cinzas }) .
$$

O conteúdo de carboidratos não-fibrosos (CNF), pela diferença entre CHOT e FDN e os teores de carboidratos não-fibrosos corrigidos para cinzas e proteína (CNFcp) foram calculados como proposto por Detmann e Valadares Filho (2010).

$$
\mathrm{CNF} p \mathrm{p}=(100-(\% \text { FDNcp }-(\% \mathrm{~PB}-\mathrm{PBu}+\mathrm{U})-\% \mathrm{EE}-\% \text { cinzas })) \text {, }
$$

em que: $\mathrm{PBu}=$ proteína bruta da ureia; $\mathrm{U}=$ quantidade de uréia.

Os nutrientes digestíveis totais (NDT) foram calculados segundo NRC (2001), utilizando-se a seguinte equação: $\mathrm{NDT}(\%)=\mathrm{CFNcp}+\mathrm{PBD}+$ $(2,25 \mathrm{xEED})+$ FDNcp -7

Em que: PBD, FDNcpD, CNFcpD e EED representam, respectivamente, ingestões de $\mathrm{PB}$, FDNcp, CNFcp e EE digestíveis.

Para a energia metabolizável, considerouse que $1,0 \mathrm{~kg}$ de NDT equivalem a 4,409 Mcal de energia digestível e para a transformação em energia metabolizável utilizou-se o valor de $82 \%$ de eficiência de utilização de energia digestível (NRC, 1996).

Para estimar a ingestão de nutrientes oriundos de suplemento utilizou-se o óxido crômico $\left(\mathrm{Cr}_{2} \mathrm{O}_{3}\right)$ e LIPE $^{\circledR}$ como indicadores externos segundo metodologias descritas por Kimura e Miller (1957) e Saliba et al. (2003), respectivamente e, a ingestão de pasto por meio da fibra insolúvel e fibra indigestível em detergente neutro (FDNi) como indicador interno Casali et al. (2008) (Tabela 2).

A glicerina utilizada foi analisada pelo método de Karl Fischer no Instituto de Tecnologia do Paraná - TECPAR, Curitiba - PR e apresentou a seguinte composição físico-química em porcentagem na matéria natural: 43,9\% de glicerol, 6,0\% de metanol, 33,6 \% de ácidos graxos totais, 9,0\% de água e 7,3\% de matéria mineral.

Os animais foram abatidos segundo rotina do frigorifico comercial e logo após o abate, as carcaças foram identificadas e, posteriormente, resfriadas por 24 horas a $2^{\circ} \mathrm{C}$. Após o resfriamento, uma seção do músculo Longissimus dorsi, entre a $11^{\mathrm{a}}$ e $13^{\mathrm{a}}$ costelas de cada meia-carcaça esquerda, foi retirada, congelada e encaminhada ao laboratório de análises de alimento e nutrição animal da Universidade Estadual de Maringá - PR para análises posteriores.

Para a extração dos lipídios totais dos concentrados, forragem e da glicerina bruta utilizou-se a metodologia proposta por Folch, Lees e Stanley (1957) e para os lipídios da carne o método desenvolvido por Bligh e Dyer (1959), fazendo-se a correção da umidade das amostras para $80 \%$.

O método utilizado para a preparação dos ésteres metílicos dos ácidos graxos foi realizada conforme método 5509 da ISO (1978).

A análise cromatográfica foi conduzida utilizando um cromatógrafo a gás Varian, modelo CP-3380, equipado com detector de ionização em chama, injetor do tipo com/sem divisão de amostra e coluna capilar de sílica fundida CP-7420 (100\% cianopropil ligado, dimensões: $100 \mathrm{~m}, 0,25 \mathrm{~mm}$ d.i. e $0,25 \mu \mathrm{m}$ de fase estacionária). 
Tabela 2. Ingestões médias diárias de nutrientes por bovinos suplementados em pastagem de Brachiaria decumbens com glicerina de baixa pureza.

\begin{tabular}{|c|c|c|c|c|c|c|c|c|c|}
\hline \multirow{2}{*}{ Itens } & \multicolumn{5}{|c|}{ Níveis de inclusão de glicerina (\%MS) } & \multirow{2}{*}{ EPM } & \multicolumn{3}{|c|}{ P Valor } \\
\hline & 0 & 3 & 6 & 9 & 12 & & $\mathrm{~L}$ & Q & $\mathrm{C}$ \\
\hline $\operatorname{MSS}^{1}(\mathrm{~kg} / \mathrm{dia})$ & 4,16 & 4,60 & 3,92 & 4,78 & 3,83 & 0,15 & 0,975 & 0,490 & 0,603 \\
\hline MSS (\%PC) & 0,90 & 1,01 & 0,81 & 1,00 & 0,80 & 0,03 & 0,789 & 0,665 & 0,799 \\
\hline $\operatorname{MSV}^{2}(\mathrm{~kg} / \mathrm{dia})$ & 7,05 & 5,51 & 6,01 & 6,4 & 5,85 & 0,21 & 0,290 & 0,341 & 0,112 \\
\hline MSV (\%PC) & 1,54 & 1,20 & 1,23 & 1,34 & 1,25 & 0,05 & 0,263 & 0,236 & 0,151 \\
\hline $\operatorname{MST}^{3}(\mathrm{~kg} / \mathrm{dia})$ & 11,21 & 10,12 & 9,94 & 11,18 & 9,68 & 0,24 & 0,363 & 0,657 & 0,150 \\
\hline MST (\%PC) & 2,45 & 2,21 & 2,04 & 2,34 & 2,06 & 0,06 & 0,296 & 0,497 & 0,311 \\
\hline $\mathrm{MO}^{4}(\mathrm{~kg} / \mathrm{dia})$ & 10,47 & 9,54 & 9,17 & 10,49 & 9,05 & 0,22 & 0,367 & 0,665 & 0,160 \\
\hline $\mathrm{FDN}^{5}(\mathrm{~kg} / \mathrm{dia})$ & 6,0 & 4,90 & 5,14 & 5,32 & 4,82 & 0,15 & 0,076 & 0,158 & 0,079 \\
\hline FDN (\%PC) & 1,31 & 1,09 & 1,05 & 1,11 & 1,03 & 0,04 & 0,090 & 0,136 & 0,131 \\
\hline $\mathrm{PB}^{6}(\mathrm{~kg} / \mathrm{dia})$ & 1,88 & 1,90 & 1,80 & 1,92 & 1,61 & 0,04 & 0,186 & 0,186 & 0,206 \\
\hline $\mathrm{EE}^{7}(\mathrm{~kg} / \mathrm{dia})$ & 0,35 & 0,40 & 0,42 & 0,51 & 0,50 & 0,01 & $<.0001 *$ & $<.0001$ & 0,0002 \\
\hline $\mathrm{CNF}^{8}(\mathrm{Kg} / \mathrm{dia})$ & 2,53 & 2,58 & 2,20 & 3,07 & 2,40 & 0,08 & 0,471 & 0,682 & 0,241 \\
\hline $\mathrm{NDT}^{9}(\mathrm{Kg} /$ dia $)$ & 6,90 & 6,30 & 5,80 & 7,13 & 5,08 & 0,24 & 0,169 & 0,337 & 0,087 \\
\hline $\mathrm{ED}^{10}$ (Mcal/dia $)$ & 30,42 & 27,80 & 25,50 & 31,45 & 22,41 & 1,08 & 0,169 & 0,337 & 0,087 \\
\hline $\mathrm{EM}^{11}(\mathrm{Mcal} / \mathrm{dia})$ & 24,94 & 22,80 & 20,91 & 25,79 & 18,37 & 0,89 & 0,169 & 0,337 & 0,087 \\
\hline
\end{tabular}

EPM=Erro padrão da média; L, Q e C: ordem dos efeitos linear, quadrático e cúbico para a inclusão da glicerina de baixa pureza na dieta; ${ }^{1}$ Matéria seca do suplemento; ${ }^{2}$ Matéria seca do volumoso $;{ }^{3}$ Matéria seca total ; ${ }^{4}$ Matéria orgânica; ${ }^{5}$ Fibra em detergente neutro; ${ }^{6}$ Proteína bruta; ${ }^{7}$ Extrato etéreo; ${ }^{8}$ Carboidratos não fibrosos; ${ }^{9}$ Nutrientes digestíveis totais; ${ }^{10}$ Energia digestível; ${ }^{11}$ Energia metabolizável. ${ }^{*} \hat{Y}=0,4239 x+0,30926\left(R^{2}=0,46\right)$.

Fonte: Elaboração dos autores.

As temperaturas do injetor e do detector foram mantidas a $220^{\circ} \mathrm{C}$ e $240^{\circ} \mathrm{C}$, respectivamente. As vazões dos gases foram de 1,4 $\mathrm{mL} \mathrm{min}^{-1}$ para o gás de arraste $\left(\mathrm{H}_{2}\right), 30 \mathrm{~mL} \mathrm{~min}^{-1}$ para o gás-auxiliar $\left(\mathrm{N}_{2}\right)$, $30 \mathrm{~mL} \mathrm{~min}^{-1}$ e $300 \mathrm{~mL} \mathrm{~min}{ }^{-1}$ para o gás $\left(\mathrm{H}_{2}\right)$ e para o ar sintético da chama, respectivamente.

O divisor de amostra foi de 1/80. Os parâmetros de operação usados foram: temperatura da coluna de $165^{\circ} \mathrm{C}$ por 12 minutos, sendo então elevada para $180^{\circ} \mathrm{C}$ a uma taxa de $40^{\circ} \mathrm{C} \mathrm{min}^{-1}$, permanecendo nessa temperatura por 15 minutos e em seguida elevada para $240^{\circ} \mathrm{C}$ a uma taxa de $15^{\circ} \mathrm{C} \mathrm{min}^{-1}$, por 18,62 minutos, que resultou em uma corrida de 50 minutos. As áreas dos picos foram determinadas através do software Workstation 5.0 (Varian).

A quantificação dos ácidos graxos foi realizada após a normalização das áreas. Os picos foram identificados por comparação dos tempos de retenção de padrões de ésteres metílicos de ácidos graxos Sigma (EUA) e após verificação do comprimento equivalente de cadeia.

O perfil dos ácidos graxos dos suplementos, da Brachiaria decumbens e da glicerina de baixa pureza encontra-se na Tabela 3 e o consumo de ácidos graxos na Tabela 5. A composição de ácidos graxos depositados no músculo Longissimus dorsi encontra-se na Tabela 6. 
Tabela 3. Perfil de ácidos graxos dos suplementos, Brachiaria decumbens e da glicerina de baixa pureza.

\begin{tabular}{|c|c|c|c|c|c|c|c|}
\hline \multirow{2}{*}{ Ácidos graxos (\%) } & \multicolumn{5}{|c|}{ Níveis de inclusão de glicerina (\%MS) } & \multirow[b]{2}{*}{ Brachiaria } & \multirow[b]{2}{*}{ Glicerina } \\
\hline & 0 & 3 & 6 & 9 & 12 & & \\
\hline \multicolumn{8}{|l|}{ Saturados (AGS) } \\
\hline $\begin{array}{l}14: 0 \\
\text { miristico }\end{array}$ & 0,3 & 0,2 & 0,2 & 0,3 & 0,3 & 1,8 & 0,8 \\
\hline $\begin{array}{l}\text { 16:0 } \\
\text { palmítico }\end{array}$ & 14,8 & 13,2 & 14,0 & 15,1 & 15,8 & 31,9 & 20,7 \\
\hline $\begin{array}{l}\text { 18:0 } \\
\text { esteárico }\end{array}$ & 2,7 & 3,3 & 2,6 & 2,6 & 2,6 & 2,1 & 5,4 \\
\hline \multicolumn{8}{|c|}{ Monoinsaturados (AGMI) } \\
\hline $\begin{array}{l}\text { 15: } 1 \omega-9 \\
\text { pentadecanóico }\end{array}$ & 0,0 & 0,0 & 0,0 & 0,0 & 0,0 & 1,3 & 0,0 \\
\hline $\begin{array}{l}16: 1 \omega-9 \\
9 \text { palmitoléico }\end{array}$ & 0,3 & 0,2 & 0,2 & 0,2 & 0,3 & 0,7 & 0,0 \\
\hline $\begin{array}{l}\text { 18:1 } \omega-9 \\
\text { oléico }\end{array}$ & 30,2 & 29,8 & 25,8 & 23,1 & 21,0 & 2,0 & 23,2 \\
\hline 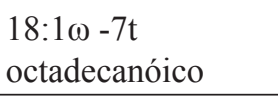 & 0,6 & 0,8 & 0,8 & 0,8 & 0,8 & 0,5 & 1,0 \\
\hline \multicolumn{8}{|c|}{ Poliinsaturados (AGPI) } \\
\hline $\begin{array}{l}18: 2 \omega-6 \\
\text { linoléico }\end{array}$ & 49,2 & 50,5 & 54,0 & 56,5 & 56,8 & 14,9 & 46,2 \\
\hline $\begin{array}{l}18: 3 \omega-3 \\
\alpha \text { linolênico }\end{array}$ & 1,9 & 2,0 & 2,3 & 2,4 & 2,4 & 44,8 & 0,6 \\
\hline $\begin{array}{l}18: 3 \omega-6 \\
\gamma \text { linolênico }\end{array}$ & 0,0 & 0,0 & 0,0 & 0,0 & 0,0 & 0,0 & 2,0 \\
\hline \multicolumn{8}{|l|}{ Somatórios e Razão } \\
\hline AGS & 17,8 & 16,7 & 16,8 & 17,0 & 18,7 & 35,8 & 27,0 \\
\hline AGMI & 31,1 & 30,8 & 26,9 & 24,1 & 22,1 & 4,5 & 24,2 \\
\hline AGPI & 51,1 & 52,5 & 56,3 & 58,9 & 59,2 & 59,7 & 48,8 \\
\hline$\omega-6^{1}$ & 49,2 & 50,5 & 54,0 & 56,5 & 56,9 & 14,9 & 48,2 \\
\hline$\omega-3^{2}$ & 1,9 & 2,0 & 2,3 & 2,4 & 2,4 & 44,8 & 0,6 \\
\hline$\omega-9^{3}$ & 30,5 & 30,0 & 26,6 & 23,9 & 21,3 & 4,0 & 23,2 \\
\hline $\mathrm{AGS} / \mathrm{AGPI}^{4}$ & 0,3 & 0,3 & 0,3 & 0,3 & 0,3 & 0,6 & 0,6 \\
\hline$\omega-6 / \omega-3^{5}$ & 25,9 & 25,3 & 23,5 & 23,5 & 23,7 & 0,3 & 80,3 \\
\hline
\end{tabular}

'ácidos graxos da série $\omega$ - 6; 2ácidos graxos da série $\omega$ - 3; 3ácidos graxos da série $\omega$ - 9; ${ }^{4}$ razão ácidos graxos saturados:ácidos graxos polinsaturados; ${ }^{5}$ razão ácidos graxos da série $\omega$ - 6 : ácidos graxos da série $\omega$ - 3 .

Fonte: Elaboração dos autores.

A significância do coeficiente de correlação foi dos ácidos graxos consumidos com a composição testada por meio do teste "t" a 5\% de probabilidade, dos ácidos graxos encontrados no músculo. Em que r utilizando o programa estatístico SAEG (2001). assume valores entre -1 (associação linear negativa) Para análise dos dados, foi calculado o coeficiente e 1 (associação linear positiva). A interpretação dos de correlação linear de Pearson entre a composição valores de r encontra-se na Tabela 4. 
Tabela 4. Interpretação dos coeficientes de correlação linear de Pearson

\begin{tabular}{cc}
\hline Valores de "r" & Interpretação \\
\hline 0,00 a 0,19 & Correlação bem fraca \\
0,20 a 0,39 & Correlação fraca \\
0,40 a 0,69 & Correlação moderada \\
0,70 a 0,89 & Correlação forte \\
0,90 a 1,00 & Correlação muito forte \\
\hline
\end{tabular}

Fonte: SHIKAMURA (2006).

Tabela 5. Consumo de ácidos graxos (kg/dia) de bovinos suplementados em pastagem Brachiaria decumbens com glicerina de baixa pureza.

\begin{tabular}{|c|c|c|c|c|c|}
\hline \multirow[b]{2}{*}{ Ácidos graxos } & \multicolumn{5}{|c|}{ Níveis de inclusão de glicerina } \\
\hline & 0 & 3 & 6 & 9 & 12 \\
\hline \multicolumn{6}{|c|}{ Saturados (AGS) } \\
\hline $14: 0$ & 0,011 & 0,009 & 0,010 & 0,010 & 0,010 \\
\hline $16: 0$ & 0,252 & 0,231 & 0,245 & 0,245 & 0,248 \\
\hline $17: 0$ & 0,003 & 0,0031 & 0,0033 & 0,0032 & 0,003 \\
\hline 18:0 & 0,024 & 0,027 & 0,028 & 0,028 & 0,029 \\
\hline $20: 0$ & 0,002 & 0,0021 & 0,0023 & 0,0023 & 0,027 \\
\hline 21:0 & 0,001 & 0,002 & 0,001 & 0,001 & 0,001 \\
\hline $22: 0$ & 0,0003 & 0,0009 & 0,0004 & 0,0004 & 0,0004 \\
\hline \multicolumn{6}{|c|}{ Moinsaturados (AGMI) } \\
\hline $15: 1 \omega-9$ & 0,008 & 0,007 & 0,008 & 0,007 & 0,007 \\
\hline $16: 1 \omega-9$ & 0,005 & 0,004 & 0,005 & 0,005 & 0,005 \\
\hline 16: $1 \omega-7$ & 0,002 & 0,002 & 0,002 & 0,002 & 0,007 \\
\hline $18: 1 \omega-9 c$ & 0,125 & 0,135 & 0,115 & 0,108 & 0,99 \\
\hline $18: 1 \omega-7 \mathrm{t}$ & 0,004 & 0,005 & 0,005 & 0,005 & 0,002 \\
\hline $20: 1 \omega-9$ & 0,004 & 0,003 & 0,004 & 0,004 & 0,004 \\
\hline \multicolumn{6}{|c|}{ Poli-insaturados (AGPI) } \\
\hline $18: 2 \omega-6$ & 0,27 & 0,31 & 0,30 & 0,32 & 0,32 \\
\hline $18: 3 \omega-3$ & 0,27 & 0,24 & 0,27 & 0,26 & 0,26 \\
\hline \multicolumn{6}{|c|}{ Somatório e Razão } \\
\hline AGS & 0,29 & 0,27 & 0,29 & 0,28 & 0,29 \\
\hline AGMI & 0,14 & 0,16 & 0,13 & 0,12 & 0,12 \\
\hline AGPI & 0,55 & 0,55 & 0,57 & 0,58 & 0,58 \\
\hline$\omega-6^{1}$ & 0,24 & 0,30 & 0,30 & 0,32 & 0,32 \\
\hline$\omega-3^{2}$ & 0,27 & 0,24 & 0,27 & 0,26 & 0,20 \\
\hline$\omega-9^{3}$ & 0,14 & 0,15 & 0,13 & 0,12 & 0,11 \\
\hline AGS:AGPI ${ }^{4}$ & 0,005 & 0,005 & 0,005 & 0,005 & 0,005 \\
\hline$\omega-6: \omega-3^{5}$ & 0,10 & 0,11 & 0,10 & 0,10 & 0,10 \\
\hline
\end{tabular}

${ }^{1}$ ácidos graxos da série $\omega-6$; ${ }^{2}$ ácidos graxos da série $\omega-3 ;{ }^{3}$ ácidos graxos da série $\omega$ - 9; ${ }^{2}$ ácidos graxos poliinsaturadso:ácidos graxos saturados; ;́́cidos graxos da série $\omega$ - 6: ácidos graxos da série $\omega-3$.

Fonte: Elaboração dos autores. 
Tabela 6. Perfil de ácidos graxos presentes no músculo Longissimus dorsi de bovinos suplementados em pastagem de Brachiaria decumbens com glicerina de baixa pureza.

\begin{tabular}{|c|c|c|c|c|c|}
\hline \multirow[b]{2}{*}{ Ácidos graxos } & \multicolumn{5}{|c|}{ Níveis de inclusão de glicerina (\%MS) } \\
\hline & 0 & 3 & 6 & 9 & 12 \\
\hline \multicolumn{6}{|l|}{ Saturados (AGS) } \\
\hline 12:0 (láurico) & 0,16 & 0,13 & 0,13 & 0,12 & 0,13 \\
\hline 13:0 (tridecanóico) & 0,13 & 0,16 & 0,11 & 0,11 & 0,11 \\
\hline 14:0 (miristico) & 2,09 & 1,74 & 2,48 & 2,41 & 2,37 \\
\hline 15:0 (pentadecanóico) & 0,43 & 0,43 & 0,43 & 0,44 & 0,47 \\
\hline 16:0 (palmitico) & 23,49 & 21,86 & 23,38 & 23,35 & 22,95 \\
\hline 17:0 (margárico) & 0,91 & 0,88 & 1,04 & 0,87 & 1,03 \\
\hline 18:0 (esteárico) & 17,96 & 17,94 & 19,98 & 16,63 & 17,06 \\
\hline 20:0 (araquidico) & 0,11 & 0,10 & 0,11 & 0,09 & 0,11 \\
\hline 21:0 (heneicosanóico) & 0,54 & 0,62 & 0,80 & 0,97 & 1,15 \\
\hline 22:0 (behênico) & 0,15 & 0,15 & 0,16 & 0,12 & 0,15 \\
\hline 24:0 (lignocérico) & 0,63 & 0,79 & 0,42 & 0,55 & 0,43 \\
\hline \multicolumn{6}{|l|}{ Monoinsaturados (AGMI) } \\
\hline 14:1 (mirístoléico) & 0,31 & 0,29 & 0,37 & 0,44 & 0,47 \\
\hline 15:1 $\omega$ - 9 (9pentadecanóico) & 0,25 & 0,25 & 0,20 & 0,17 & 1,18 \\
\hline 16:1 $\omega-7$ (7 palmitoléico) & 0,55 & 0,52 & 0,58 & 0,48 & 0,53 \\
\hline 16:1 $\omega 9$ (9 palmitoléico) & 2,37 & 2,13 & 2,16 & 2,53 & 2,53 \\
\hline 17:1 $\omega-9$ (9 heptadecanóico) & 0,68 & 0,65 & 0,62 & 0,67 & 0,73 \\
\hline 18:1 $\omega-7 c$ (cis vacênico) & 1,15 & 1,14 & 1,00 & 1,10 & 1,11 \\
\hline 18:1 $\omega-9$ (oléico) & 32,47 & 31,01 & 32,25 & 30,99 & 33,36 \\
\hline 18:1 $t-11$ (trans vacênico) & 2,00 & 2,50 & 4,13 & 4,37 & 5,54 \\
\hline \multicolumn{6}{|l|}{ Poli-insaturados (AGPI) } \\
\hline 18:2 $\omega$ - 6 (linoléico) & 8,65 & 9,02 & 5,54 & 9,14 & 6,47 \\
\hline $18: 2 c-9, t-11(\mathrm{CLA})$ & 0,08 & 0,08 & 0,10 & 0,08 & 0,10 \\
\hline 18:3 $\omega-69(\gamma$ linolênico $)$ & 0,14 & 0,13 & 0,14 & 0,14 & 0,15 \\
\hline 18:3 $\omega-3(\alpha$ linolênico $)$ & 1,27 & 1,42 & 0,83 & 1,07 & 0,79 \\
\hline 20:2 $\omega-6$ (eicosadienóico) & 0,04 & 0,03 & 0,01 & 0,01 & 0,01 \\
\hline 20:4 $\omega-6$ (araquidônico) & 0,29 & 0,27 & 0,17 & 0,23 & 0,17 \\
\hline $20: 3 \omega-3$ (eicosatrienóico) & 0,14 & 0,15 & 0,11 & 0,11 & 0,09 \\
\hline $20: 5 \omega-3$ (eicosapentaenoico) & 0,12 & 0,13 & 0,10 & 0,11 & 0,08 \\
\hline 22:6 $\omega-3$ (docosahexanóico) & 2,74 & 3,23 & 1,66 & 2,57 & 1,61 \\
\hline \multicolumn{6}{|l|}{ Somatório e Razão } \\
\hline AGS & 46,69 & 44,85 & 49,09 & 45,71 & 46,01 \\
\hline AGMI & 39,81 & 38,52 & 41,34 & 40,78 & 44,48 \\
\hline AGPI & 13,40 & 16,53 & 9,45 & 13,41 & 9,39 \\
\hline$\omega-6^{1}$ & 9,12 & 11,58 & 6,75 & 9,54 & 6,82 \\
\hline$\omega-3^{2}$ & 4,28 & 4,95 & 2,70 & 3,87 & 2,57 \\
\hline AGS:AGPI ${ }^{3}$ & 3,5 & 2,7 & 5,2 & 3,4 & 4,9 \\
\hline$\omega-6: \omega-3^{4}$ & 2,21 & 2,32 & 2,51 & 2,52 & 2,67 \\
\hline
\end{tabular}

1ácidos graxos da série 6 - $\omega ;{ }^{2}$ ácidos graxos da série $\omega-3 ;{ }^{3}$ razão ácidos graxos saturados : ácidos graxos polinsaturados; ${ }^{4}$ razão ácidos graxos da série $\omega$ - 6: ácidos graxos da série $\omega-3$.

Fonte: Elaboração dos autores. 


\section{Resultados e Discussão}

O ácido graxo (AG) esteárico (18:0) da dieta apresentou correlação linear positiva moderada com o AG heneicosanóico (21:0) encontrado na carne (Tabela 7). Essa correlação não tem uma explicação bem definida, pois os ácidos graxos de cadeia ímpar são formados pela síntese de novo, a partir do propionato resultante da fermentação ruminal, que pela ação da propionil-CoA sintetase, encontrada nas bactérias, é transformado em propionil-CoA e finalmente a AG de cadeia ímpar (FERNANDES; SAMPAIO; HENRIQUE, 2009).

$\mathrm{O}$ ácido heneicosanóico, ainda que seja um ácido graxo saturado (AGS) não tem efeito sobre o nível de lipoproteína de baixa densidade (LDL) plasmático, pois sendo um AG de cadeia longa apresenta baixa absorção intestinal (PALMQUIST; MATTOS, 2006).

Correlação positiva forte foi observada entre o AG esteárico e o AG trans vacênico (18:1 $t-$ 11). O AG esteárico pode ter inibido o processo de biohidrogenação do ácido trans vacênico a ácido graxo linoleico conjugado (CLA) no rúmen, favorecendo a absorção intestinal do ácido trans vacênico e consequentemente sua deposição no músculo.

Tabela 7. Correlação entre ácidos graxos da dieta e os ácidos graxos presentes no músculo Longissimus dorsi de bovinos suplementados em pastagem de Brachiaria decumbens com glicerina de baixa pureza.

\begin{tabular}{|c|c|c|c|c|c|c|c|c|}
\hline \multirow{3}{*}{$\begin{array}{l}\text { Ácidos graxos } \\
\text { da carne }\end{array}$} & \multicolumn{8}{|c|}{ Ácidos graxos da dieta } \\
\hline & \multicolumn{2}{|c|}{$18: 0$} & \multicolumn{2}{|c|}{$18: 1 \omega-9$} & \multicolumn{2}{|c|}{$18: 1 \omega-7$} & \multicolumn{2}{|c|}{$18: 2 \omega-6$} \\
\hline & $\mathrm{r}$ & $\mathrm{P}$ & $\mathrm{r}$ & $\mathrm{P}$ & $\mathrm{r}$ & $\mathrm{P}$ & $\mathrm{r}$ & $\mathrm{P}$ \\
\hline $21: 0$ & 0,65 & $<0,0001$ & $-0,50$ & 0,002 & $-0,58$ & 0,002 & - & - \\
\hline $18: 1 t-11$ & 0,73 & $<0,0001$ & - & - & $-0,54$ & 0,0006 & 0,38 & 0,027 \\
\hline $18: 2 c-9, t-11$ & - & - & $-0,35$ & 0,037 & - & - & - & - \\
\hline $18: 3 \omega-3$ & $-0,35$ & 0,034 & $-0,38$ & 0,022 & 0,33 & 0,046 & - & - \\
\hline $20: 2 \omega-6$ & - & - & 0,52 & 0,0013 & - & - & - & - \\
\hline $20: 3 \omega-3$ & $-0,37$ & 0,025 & - & - & $-0,37$ & 0,027 & - & - \\
\hline $20: 5 \omega-3$ & $-0,33$ & 0,046 & - & - & 0,36 & 0,029 & - & - \\
\hline $\mathrm{AGMI}^{1}$ & - & - & $-0,37$ & 0,025 & $-0,53$ & 0,0009 & - & - \\
\hline$\omega-6: \omega-3$ & 0,52 & 0,001 & - & - & & & 0,33 & 0,048 \\
\hline
\end{tabular}

${ }^{1}$ ácidos graxos monoinsaturados.

Fonte: Elaboração dos autores.

Em seres humanos, por proporcionarem aumento na concentração de LDL, os ácidos trans são considerados perigosos para a saúde. Partículas de LDL causam lesão no endotélio vascular com o desenvolvimento de doença inflamatória crônica da camada íntima de artérias de médio e grande calibre chamada de ateroesclerose (SBC, 2007).

O AG esteárico apresentou correlação negativa fraca com os ácidos graxos $\alpha$ linolênico (18:3 $\omega$-3), eicosatrienóico (20:3 $\omega$-3) e o eicosapentaenoico - EPA (20:5 $\omega$-3) e correlação positiva moderada com a razão entre os ácidos graxos da série $\omega$ - 6 e $\omega$ - 3 (Tabela 7). Segundo Gregory et al. (2009) o AG $\alpha$ linolênico é convertido, no tecido adiposo, nos demais ácidos graxos da série $\omega-3$, assim a diminuição da concentração do AG $\alpha$ linolênico pode ter promovido déficit na síntese dos ácidos graxos eicosatreinóico e EPA e por conseguinte aumento na razão $\omega-6: \omega$ - 3 na carne. 


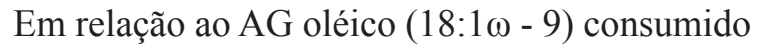
constatou-se correlação negativa moderada com os ácidos graxos heneicosanóico (21:0), e negativa fraca com os ácidos CLA (18:2 c - 9, $t-11), \alpha$ linolênico (18:3 $\omega$ - 3) e com AGMI e correlação positiva moderada com o AG eicosadienóico (20:2 $\omega$ - 6) do músculo (Tabela 7).

O AG oléico pode competir com o AG $\alpha$ linolênico, bioprecursor dos ácidos graxos da série $\omega-3$, e com seus produtos intermediários pelas enzimas dessaturases e elongases (WOUTERSEN et al., 1999). Isso pode ter sido a razão da diminuição da deposição do AG $\alpha$ linolênico e do CLA na carne e consequentemente, redução na concentração do somatório dos ácidos graxos monoinsaturados (AGMI).

OAG cis vacênico $(18: 1 \omega-7)$ da dieta apresentou correlação negativa moderada com os ácidos graxos heneicosanóico (21:0), trans vacênico (18:1t-11) e AGMI e negativa fraca com os ácidos graxos $\alpha$ linolênico (18:3 $\omega-3)$, eicosatrienóico (20:3 $\omega$-3) e com o EPA. Esses resultados demonstram que a presença do AG cis vacênico pode ter favorecido a deposição dos ácidos da série $\omega$ - 3 no músculo Longissimus dorsi.

O aumento do teor $\omega$ - 3 na carne pode influenciar a cor, reduzir o tempo de prateleira e melhorar os atributos sensoriais da carne (SCOLLAN et al., 2006). Por outro lado, os ácidos graxos da série $\omega$ - 3 podem diminuir a síntese da lipoproteína de baixíssima densidade (VLDL), as concentrações de triglicerídeos, a adesividade plaquetária e promoverem pequena redução na pressão arterial bem como o risco de doença coronária (OLIVEIRA; VIEIRA, 2004; RIQUE; SOARES; MEIRELLES, 2002).

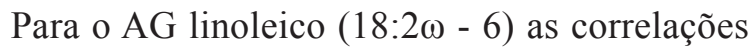
foram positivas fracas com os ácidos trans vacênico (18:1-11t) e com a razão $\omega-6$ : $\omega-3$. O AG linoleico está presente em maior quantidade no alimento concentrado e na glicerina do que na forragem, como demonstrado na Tabela 3, isso pode ter refletido no aumento dos teores do seu isômero resultante da biohidrogenação, o ácido trans vacênico.

O AGMI e o somatório dos ácidos graxo polinsaturados (AGPI) apresentaram correlação positiva com a maioria dos ácidos graxos da carne (Tabela 8). O consumo de AGMI pode proporcionar elevação nos níveis de ácidos graxos da série $\omega-3$ e $\omega-6$, AGMI, AGPI e da razão entre AGPI : AGS na carne.A ingestão de AGPI pode promover aumento do AG trans vacênico e do CLA.

Os AGMI e principalmente, os AGPI proporcionam benefícios à saúde humana (SCOLLAN et al., 2006), pois promovem diminuição do colesterol total e de LDL e aumento dos níveis de HDL no plasma. Segundo Metz et al. (1997), as maiores organizações de saúde dos Estados Unidos têm proposto a proporção de 2:1,5 respectivamente para AGMI e AGPI, na prevenção e tratamento de dislipidemias, e hipertensão, porém nesse estudo a proporção media alcançada foi de 0,27:1 (Tabela 8). 
Tabela 8. Correlação entre os somatórios dos ácidos graxo insaturados da dieta com os ácidos graxos do músculo Longissimus dorsi de bovinos suplementados em pastagem de Brachiaria decumbens com glicerina de baixa pureza.

\begin{tabular}{|c|c|c|c|c|c|c|c|c|}
\hline \multirow{3}{*}{$\begin{array}{l}\text { Ácidos graxos da } \\
\text { carne }\end{array}$} & \multicolumn{8}{|c|}{ Ácidos graxos poliinsaturados da dieta } \\
\hline & \multicolumn{2}{|c|}{ AGMI } & \multicolumn{2}{|c|}{ AGPI } & \multicolumn{2}{|c|}{$\omega-6$} & \multicolumn{2}{|c|}{$\omega-9$} \\
\hline & $\mathrm{r}$ & $\mathrm{P}$ & $\mathrm{r}$ & $\mathrm{P}$ & $\mathrm{r}$ & $\mathrm{P}$ & $\mathrm{r}$ & $\mathrm{P}$ \\
\hline 21:0 & $-0,66$ & $<0,0001$ & 0,88 & $<0,0001$ & 0,40 & 0,017 & 0,64 & $<0,0001$ \\
\hline $24: 0$ & $-0,42$ & 0,010 & 0,44 & 0,006 & - & - & 0,42 & 0,011 \\
\hline $18: 1 \omega t-11$ & $-0,55$ & 0,0005 & 0,87 & $<0,001$ & 0,51 & 0,001 & 0,53 & 0,009 \\
\hline $18: 2 c-9, t-11$ & $-0,40$ & 0,014 & 0,39 & 0,019 & - & - & 0,40 & 0,15 \\
\hline $18: 3 \omega-6$ & 0,37 & 0,026 & - & - & - & - & 0,37 & 0,28 \\
\hline $18: 3 \omega-3$ & 0,42 & 0,11 & $-0,54$ & 0,0008 & - & - & 0,41 & 0,013 \\
\hline $20: 2 \omega-6$ & 0,45 & 0,006 & $-0,41$ & 0,012 & - & - & 0,45 & 0,05 \\
\hline $20: 3 \omega-3$ & 0,38 & 0,21 & $-0,52$ & 0,001 & - & - & 0,37 & 0,26 \\
\hline $22: 6 \omega-3$ & 0,39 & 0,018 & $-0,44$ & 0,007 & - & - & 0,38 & 0,21 \\
\hline $\mathrm{AGMI}^{1}$ & $-0,47$ & 0,003 & 0,53 & 0,0009 & - & - & 0,45 & 0,05 \\
\hline $\mathrm{AGPI}^{2}$ & 0,40 & 0,017 & $-0,41$ & 0,013 & - & - & 0,39 & 0,020 \\
\hline$\omega-6^{3}$ & 0,38 & 0,021 & 0,37 & 0,026 & - & - & 0,38 & 024 \\
\hline$\omega-3^{4}$ & 0,41 & 0,013 & $-0,48$ & 0,003 & - & - & 0,40 & 0,16 \\
\hline AGPI:AGS 5 & 0,35 & 0,036 & $-0,35$ & 0,035 & - & - & 0,34 & 0,039 \\
\hline$\omega-6: \omega-3^{6}$ & - & - & 0,57 & 0,0003 & 0,42 & 0,001 & - & - \\
\hline
\end{tabular}

${ }^{1}$ ácidos graxos monoinsaturados; ${ }^{2}$ ácidos graxos poliinsaturados; ${ }^{3}$ ácidos graxos da série $\omega-6 ;{ }^{4}$ ácidos graxos da série $\omega$ - 3 ; 5ácidos graxos poliinsaturados:ácidos graxos saturados; ${ }^{6}$ ácidos graxos de série $\omega$ - 6: ácidos graxos da série $\omega$ - 3 .

Fonte: Elaboração dos autores.

Os ácidos graxos da série $\omega-6$ apresentaram correlação positiva com o AG henicosanóico e com a razão $\omega$ - 6: $\omega$ - 3. Os da série $\omega-9$ apresentaram maior número de correlações, sendo assim distribuídas em positiva com os ácidos graxos lignocérico (24:0), $\alpha$

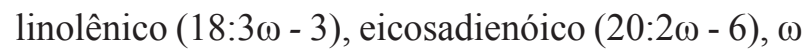

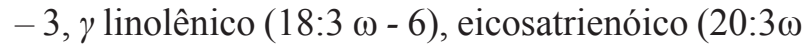
- 3), docosahexanóico (22:6 $\omega$ - 3), AGPI, $\omega$ - 6 e com AGPS : AGP e negativa com os ácidos graxos heneicosanóico, trans vacênico (18:1 $\omega$-11t) e com o AGMI (Tabela 8).

SegundoFarfan(1996), osácidos graxos saturados que apresentam efeito hipercolesterolêmico são os ácidos graxos láurico, mirístico e palmítico. Neste estudo não foram encontradas nenhuma correlação entre os ácidos graxos da dieta com esses ácidos graxos saturados.

\section{Conclusões}

Os ácidos graxos presentes nas dietas com teores de inclusão de glicerina de baixa pureza apresentaram correlações com a maioria dos ácidos graxos depositados no músculo Longissimus dorsi e alteraram o perfil dos mesmos, reduziu a deposição dos ácidos graxos monoinsaturados e aumentou os da série $\omega-6$, assim como a razão entre os ácidos graxos da série $\omega-6$ e os da série $\omega-3$, mas não modificaram a deposição dos ácidos graxos láurico, mirístico e palmítico, considerados como hipercolesterolêmicos.

\section{Agradecimentos}

A UFRB/ Campus de Cruz das Almas, BA, a UESB/Campus de Itapetinga, BA e a FAPESB 


\section{Referências}

BLIGH, E. G.; DYER, W. J. A rapid method of total lipid extraction and purification. Canadian Journal of Biochemistry and Physiology, Ottawa, v. 37, n. 8, p. 911917, 1959.

BROUWER. I. A.; WANDER, S. A.; KATAN, M. B. Trans fatty acids and cardiovascular health: research completed? Kiel: European Journal of Clinical Nutrition, 2013. Available at: <http://www.ncbi.nlm.nih.gov/ pubmed/23531781>. Accessed at: 10 apr. 2013.

CASALI, A. O.; DETMANN, E.; VALADARES FILHO, S. C.; PEREIRA, J. C.; HENRIQUES, L. T. FREITAS, S.G.; PAULINO, M. F. Influência do tempo de incubaçãoe do tamanho de partículas sobre os teores de compostosindigestíveis em alimentos e fezes bovinas obtidos por procedimentos in situ. Revista Brasileira de Zootecnia, Viçosa, v. 37, n. 2, p. 335-342, 2008.

COSTA, R. G.; CARTAXO, F. Q.; SANTOS, N. M.; QUEIROGA, R. C. R. E. Carne caprina e ovina: composição lipídica e características sensoriais. Revista Brasileira de Saúde e Produção Animal, Salvador, v. 9, n. 3, p. 497-506, 2008.

DEFRAIN, J. M.; HIPPEN, R.; KALSCHEUR, K. F.; JARDON, P. W. Feeding glycerol to transition dairy cows: effects on blood metabolites and lactation performance. Journal of Dairy Science, Madison, v. 87, n. 12, p. $4195-$ 4206, 2004.

DEPARTMENT OF HEALTH. Nutritional aspects of cardiovascular disease. London: HMSO, 1994. 178 p.

DETMANN, E.; VALADARES FILHO, S. C. Sobre a estimação de carboidratos não fibrosos em alimentos e dietas. Arquivo Brasileiro de Medicina Veterinária e Zootecnia, Belo Horizonte, v. 62, n. 4, p. 980-984, 2010.

EIRAS, C. E.; BARBOSA, L. P.; MARQUES, J. A.; ARAÚJO, F. L.; LIMA, B. S.; ZAWADZKI, F.; PEROTTO, D.; PRADO, I. N. Glycerine levels in the diets of crossbred bulls finished in feedlot: animal performance, carcass dressing, feed intake and apparent digestibility. Animal Feed Science and Technology, Philadelphia, v. 179, n. 2, p. 222-226, 2014.

FARFAN, J.A. Alimentos que influenciam os níveis de colesterol no organismo. In: Instituto de Tecnologia de Alimentos. Seminário colesterol: análise, ocorrência, redução em alimentos e implicações na saúde. Campinas: ITAL, 1996. p. 35-44.

FERNANDES, A. R. M.; SAMPAIO, A. A. M.; HENRIQUE, W. Composição química e perfil de ácidos graxos da carne de bovinos de diferentes condições sexuais recebendo silagem de milho e concentrado ou cana-de-açúcar e concentrado contendo grãos de girassol. Revista Brasileira de Zootecnia, Viçosa, MG, v. 38, n. 4, p. 705-712, 2009.

FOLCH, J.; LEES, M.; STANLEY, G. H. S; A simple method for the isolation and purification of total lipids from animal tissues. Journal of Biological Chemistry, Auburn, v. 226, n. 1, p. 497-509, 1957.

GEAY, Y.; BAUCHART, D.; HOCQUETTE, J.; CULIOLI, J. Effect of nutritional factors on biochemical, structural and metabolic characteristics of muscles in ruminants, consequences on dietetic value and sensorial qualities of meat. Reproduction Nutrition Development, Paris, v. 41, n. 4, p. 1-26, 2001.

GEBAUER, S. K.; CHARDIGNY, J. M.; JAKOBSEN, M. V.; LAMARCHE, B.; LOCK, A. L.; PROCTOR, S. D.; BAER, D. J. Effects of ruminant trans fatty acids on cardiovascular disease and cancer: a comprehensive review of epidemiological, clinical, and mechanistic studies. Advances in Nutrition, Colorado, v. 2, n. 4, p. 332-54, 2011.

GREGORY, R. M.; CARDONA, J. C. A.; OSPINA, H. P.; RAMIREZ, M. H.; MATTOS, R. C.; JOBIM. M. I. M. Ácidos graxos poliinsaturados e seus efeitos no desempenho reprodutivo da vaca. Revista Brasileira de Reprodução Animal, Belo Horizonte, n. 6, p. 153-156, 2009. Suplemento.

GRUNDY, S. M.; DENKE, M. A. Dietary influences on serum lipids. The Journal of Lipid Research, Durham, v. 31, n. 7, p. 1149-1172, 1990.

GUNN, P. J.; NEARY, M. K.; LEMENAGER, R. P.; LAKE, S. L. Effects of crude glycerin on performance and carcass characteristics of finishing wether lambs. Jornal Animal Science, Champaign, v. 88, n. 5, p. 17711776, 2010.

INTERNATIONAL ORGANIZATION FOR STANDARDIZATION - ISO. Animal and vegetable fats and oils preparation of methyl esters of fatty acids. ISO(Method ISO 5509). Geneve: ISO, 1978. p. 1-6.

KIMURA, F. T.; MILlER, V. L. Chromic oxide measurement, improved determination of chromic oxide in cow feed and feces. Journal of Agriculture and Food Chemistry, Washington, v. 5, n. 3, p. 216, 1957.

LAGE, J. F.; PAULINO, P. V. R.; PEREIRA, L. G. R.; DE CAMPOS VALADARES FILHO, S.; OLIVEIRA, A. S. de; DETMANN, E.; DE PAIVA SOUZA, N. K.; LIMA, J. C. M. Glicerina bruta na dieta de cordeiros terminados em confinamento. Pesquisa Agropecuária Brasileira, Brasília, v. 45, n. 9, p. 1012-1020, 2010. 
LAMMERS, P. J.; KERR, B. J.; WEBER, T. E.; DOZIER, W. A.; KIDD, M. T.; BREGENDAHL, K.; HONEYMAN, M. S. Digestible and metabolizable energy of crude glycerol for growing pigs. Journal of Animal Science, Champaign, v. 86, n. 3, p. 602-608, 2008.

MACH, N.; BACH, A.; DEVANT, M. Effects of crude glycerin supplementation on performance and meat quality of Holstein bulls fed high-concentrate diets. Journal Animal Science, Champaign, v. 87, n. 2, p. 632638, 2009.

METZ, D. A.; KRIS-ETHERTON, P. M.; MORRIS, C. D.; MUSTAD, V. A.; STERN, J. S.; OPARIL, S.; CHAIT, A.; HAYNES, R. B.; RESNICK, L. M,.; CLARK, S.; HATTON, D. C.; MCMAHON, M.; HOLCOMB, S.; SNYDER, G. W.; PI-SUNYER, F. X.; MCCARRON, D. A. Dietary compliance and cardiovascular risk reduction with a prepared meal plan compared with a self-selected diet. American Journal Clinical of Nutrition, Bethesda, v. 66, n. 2, p. 373-385, 1997.

NATIONAL RESEARCH COUNCIL - NRC. Nutrients requeriments of beef cattle. $7^{\text {th }}$ ed. Washington: National Academy Press, 1996. 244 p.

$7^{\text {th }}$ ed. Washington, D. C., 2001. 381 p.

$7^{\text {th }}$ ed. Washington: National Academy

Press, $2000.450 \mathrm{p}$.

OLIVEIRA, J. S.; ANTONIASSI, R.; FREITAS, S. C.; MULLER, M. D. Composição química da glicerina produzida por usinas de biodiesel no Brasil e potencial de uso na alimentação animal. Ciência Rural, Santa Maria, v. 43, n. 3, p. 509-512, 2013.

OLIVEIRA, M. F.; VIEIRA, O. V. Extração de óleos de girassol utilizando miniprensa. Londrina: EMBRAPA, 2004. 30 p. (Documentos, n. 237).

PALMQUIST, D. L.; MATTOS, W. R. S. Metabolismo de lipídeos. In: BERCHIELLI, T. T. Nutrição de ruminantes. Jaboticabal: FUNEP, 2006. p. 287-310.

PARSONS, G. L.; SHELOR, M. K.; DROUILLARD, J. S. Performance and carcass traits of finishing heifers fed crude glycerin. Journal of Animal Science, Champaign, v. 87, n. 2 , p. $653-657,2008$.

RIQUE, A. B. R.; SOARES, E. A.; MEIRELLES, C. M. Nutrição e exercício na prevenção e controle das doenças cardiovasculares. Revista Brasileira de Medicina do Esporte, Niterói, v. 8, n. 6, p. 244-254, 2002.
SALIBA, E. O. S.; FERREIRA, W. M.; PEREIRA, R. A. N. Lignin from Eucalyptus grandis as indicator forrabbits in digestibility trials. Tropical e Subtropical Agroecosystems, Mérida, v. 3, n. 1, p. 107-109, 2003.

SCOLLAN, N.; HOCQUETTE, J. F.; NUERNBERG, K.; DANNERNBERGER, D.; RICHARDSON, I.; MOLONEY, A. Innovations in beef production systems that enhance the nutritional and health value of beef lipids and their relationship with meat quality. Meat Science, Champaign, v. 74, n. 1, p. 17-33, 2006.

SHIKAMURA, S. E. Estatística II. Curitiba: Departamento de Estatística, UFPR, 2006. Disponível em: $\quad<$ http://leg.ufpr.br/ silvia/CE003/node74.html $>$. Acesso em: 31 mar. 2013.

SILVA, D. J.; QUEIROZ, A. C. Análise de alimentos: métodos químicos e biológicos. 3. ed. Viçosa: UFV, 2002. $235 \mathrm{p}$.

SNIFFEN, C. J.; O'CONNOR, J. D.; VAN SOEST, P. J.; FOX, D. G.; RUSSEL, J. B. A net carbohydrate and protein system for evaluatingcattle diets: II. Carbohydrate and protein availability. Journal of Animal Science, Champaign, v. 70, n. 11, p. 3562-3577, 1992.

SOCIEDADE BRASILEIRA DE CARDIOLOGIA - SBC. IV Diretriz Brasileira sobre dislipidemias e diretriz de prevenção da aterosclerose do departamento de aterosclerose da Sociedade Brasileira de Cardiologia. Rio de Janeiro: Arquivo Brasileiro de Cardiologia, 2007. $7 \mathrm{p}$.

VAN SOEST, P. J.; ROBERTSON, J. B.; LEWIS, B. A. Symposium: carboydrate metodology, metabolism, and nutritional implications in dairy cattle. Journal of Dairy Science, Madison, v. 74, n. 10, p. 3583-3597, 1991.

WANG, C.; LIU, Q.; HUO, W. J.; YANG, W. Z.; DONG, K, H.; HUANG, Y. X.; GUO, G. Effects of glycerol on rumen fermentation, urinary excretion of purine derivatives and feed digestibility in steers. Livestock Science, Amsterdam, v. 121, n. 1, p. 15-20, 2009.

WOUTERSEN, R. A.; APPEL, M. J.; GARDERENHOETMER, A.; VAN WIJNANDS, M. V. W. Dietary fat and carcinogenesis. Mutation Research, Amsterdam, v. 443, n. 2, p. 111-127, 1999. 
